\title{
MEASURING TIDES AND BINARY PARAMETERS FROM GRAVITATIONAL WAVE DATA AND ECLIPSING TIMINGS OF DETACHED WHITE DWARF BINARIES
}

\author{
Sweta Shah ${ }^{1,2}$ and Gijs Nelemans ${ }^{1,2,3}$ \\ ${ }^{1}$ Department of Astrophysics/IMAPP, Radboud University Nijmegen, P.O. Box 9010, 6500 GL Nijmegen, The Netherlands; s.shah@ astro.ru.nl \\ ${ }^{2}$ Nikhef National Institute for Subatomic Physics, Science Park 105, 1098 XG Amsterdam, The Netherlands \\ ${ }^{3}$ Institute for Astronomy, KU Leuven, Celestijnenlaan 200D, B-3001 Leuven, Belgium \\ Received 2014 May 9; accepted 2014 June 11; published 2014 July 29
}

\begin{abstract}
The discovery of the most compact detached white dwarf (WD) binary SDSS J065133.33+284423.3 has been discussed in terms of probing the tidal effects in WDs. This system is also a verification source for the space-based gravitational wave (GW) detector, eLISA, or the evolved Laser Interferometer Space Antenna, which will observe short-period compact Galactic binaries with $P_{\text {orb }} \lesssim 5 \mathrm{hr}$. We address the prospects of performing tidal studies using eLISA binaries by showing the fractional uncertainties in the orbital decay rate, $\dot{f}$, and the rate of that decay, $\ddot{f}$ expected from both the GW and electromagnetic (EM) data for some of the high- $f$ binaries. We find that $\dot{f}$ and $\ddot{f}$ can be measured using GW data only for the most massive WD binaries observed at high frequencies. From timing the eclipses for $\sim 10 \mathrm{yr}$, we find that $\dot{f}$ can be known to $\sim 0.1 \%$ for J0651. We find that from GW data alone, measuring the effects of tides in binaries is (almost) impossible. We also investigate the improvement in the knowledge of the binary parameters by combining the GW amplitude and inclination with EM data with and without $\dot{f}$. In our previous work, we found that EM data on distance constrained the $2 \sigma$ uncertainty in chirp mass to $15 \%-25 \%$ whereas adding $\dot{f}$ reduces it to $0.11 \%$. EM data on $\dot{f}$ also constrain the $2 \sigma$ uncertainty in distance to $35 \%-19 \%$. EM data on primary mass constrain the secondary mass $m_{2}$ to factors of two to $\sim 40 \%$ whereas adding $\dot{f}$ reduces this to $25 \%$. Finally, using single-line spectroscopic data constrains $2 \sigma$ uncertainties in both the $m_{2}, d$ to factors of two to $\sim 40 \%$. Adding EM data on $\dot{f}$ reduces these $2 \sigma$ uncertainties to $\leqslant 25 \%$ and $6 \%-19 \%$, respectively. Thus we find that EM measurements of $\dot{f}$ and radial velocity are valuable in constraining eLISA binary parameters.
\end{abstract}

Key words: binaries: close - binaries: eclipsing - gravitational waves - stars: fundamental parameters white dwarfs

Online-only material: color figures

\section{INTRODUCTION}

The discovery of a detached white dwarf (WD) eclipsing binary system, SDSS J065133.33+284423.3 (hereafter J0651; Brown et al. 2011), has generated a number of discussions on the subject of tidal physics of compact objects (e.g., Piro 2011; Fuller \& Lai 2012, 2013; Burkart et al. 2013; Dall'Osso \& Rossi 2013). Its small orbital period of $P_{\text {orb }} \approx 765 \mathrm{~s}$ and the compact nature of the stars that are not yet transferring mass make it one of the most interesting candidates for studying the level of tidal interactions between the components as well as the possible astrophysical implications for WDs. J0651 is also a verification binary for the evolved Laser Interferometer Space Antenna (eLISA) ${ }^{4}$ (Amaro-Seoane et al. 2013), radiating gravitational waves $(\mathrm{GW})$ at $f=2.6 \mathrm{mHz}$ with an estimated signal-to-noise ratio $(\mathrm{S} / \mathrm{N})$ of $\sim 10$ for an observation length of two years. In this paper, we investigate detectability of the tidal effects from the GW and EM data and their implications on the astrophysical knowledge of the WDs in binary and similar systems. In addition, we discuss the effect of using measured EM period changes on the GW parameter estimates.

eLISA will observe Galactic binaries with periods shorter than a few hours. While the majority of the binaries (which are mostly double WD objects) are radiating GWs in the lowfrequency range $(f \leqslant 3 \mathrm{mHz})$, there are a handful of highfrequency sources with significant orbital decay as predicted

\footnotetext{
4 eLISA is a space-based gravitational wave mission with an expected launch of 2034.
}

by population synthesis simulations (Nelemans et al. 2004). Despite the limited number of such high- $f$ objects, they present a unique opportunity to do tidal studies of compact objects as these relatively high- $f$ binaries will have a strong gravitational signal strength and larger values for their rate of change of the orbital periods, both aiding accurate GW measurements of their orbital parameters. Here, we use Fisher studies (Cutler 1998) to address the detectability of the rate of change of the source's GW frequency, $\dot{f}$, and $\ddot{f}$ from the GW data for the detached J0651-like binary systems. The GW parameters, $f$, $\dot{f}$, and $\ddot{f}$ of a circular binary are trivially related to the more familiar quantities in EM observations, $P_{\text {orb }}, \dot{P}_{\text {orb }}$, and $\ddot{P}_{\text {orb }}$ via: $f=2 / P_{\text {orb }}, \dot{f}=-2 \dot{P}_{\text {orb }} / P_{\text {orb }}^{2}, \ddot{f}=2\left(\dot{P}_{\text {orb }}^{2}-2 P_{\text {orb }} \ddot{P}_{\text {orb }}\right) / P_{\text {orb }}^{3}$.

As a compact binary ages via $\mathrm{GW}$ dissipation, the orbital period changes as a result of increasing $\dot{f}$. If the stellar components in the binary are close enough to each other, an additional source of dissipation of orbital energy can ensue through tides and this may be reflected in its GW phase shift. In this paper, we consider only detached WD systems where both the GW emission and tidal torque (including dynamical tides) can enhance the orbital decay rate. The orbital evolution in the presence of mass transfer and GW (see Equation (12) in Nelemans et al. 2004) competes with dissipations from the tides. In these cases, their orbital evolution can be influenced by short-term variations like nova explosions and this could dramatically increase $\dot{f}, \ddot{f}$. This means that in the millions of binaries that eLISA will observe, if a number of such (masstransferring) systems undergo such orbital perturbation, their $\dot{f}, \ddot{f}$ will increase by orders of magnitude, making it possible 
Table 1

GW Parameter Values of the J0651 High-mass and High- $f$ Binary Systems

\begin{tabular}{lcccccccccc}
\hline \hline & $\mathcal{A}\left[\times 10^{-22}\right]$ & $\begin{array}{c}\phi_{0} \\
(\mathrm{rad})\end{array}$ & $\cos \iota$ & $\begin{array}{c}f\left[\times 10^{-3}\right] \\
(\mathrm{Hz})\end{array}$ & $\begin{array}{c}\dot{f} \\
\left(\mathrm{~Hz} \mathrm{~s}^{-1}\right)\end{array}$ & $\begin{array}{c}\ddot{f} \\
\left(\mathrm{~Hz} \mathrm{~s}^{-2}\right)\end{array}$ & $\begin{array}{c}\psi \\
(\mathrm{rad})\end{array}$ & $\begin{array}{c}\sin \beta \\
(\mathrm{rad})\end{array}$ \\
\hline J0651 & $1.67^{\mathrm{a}}$ & $\pi$ & 0.007 & 2.61 & $-3.35 \times 10^{-17}$ & $1.57 \times 10^{-31}$ & $\pi / 2$ & 0.101 & 1.77 & $\sim 13^{\mathrm{a}}$ \\
High-mass & $6.71^{\mathrm{b}}$ & $\pi$ & 0.007 & 2.61 & $-1.07 \times 10^{-16}$ & $1.61 \times 10^{-29}$ & $\pi / 2$ & 0.101 & 1.77 & $\sim 50^{\mathrm{b}}$ \\
${\text { High- } f^{\mathrm{c}}}^{3.69}$ & 5.41 & -0.86 & 17.69 & $-1.99 \times 10^{-13}$ & $8.19 \times 10^{-23}$ & 0.75 & 0.94 & 1.97 & $\sim 135$ \\
\hline
\end{tabular}

Notes.

${ }^{\mathrm{a}}$ For $m_{1}=0.25 M_{\odot}, m_{2}=0.55 M_{\odot}$, and $d=1.0 \mathrm{kpc}$.

${ }^{\mathrm{b}}$ High-mass system with $m_{1}, m_{2}=0.8 M_{\odot}, d=1.0 \mathrm{kpc}$.

${ }^{\mathrm{c}}$ For the given $f, \dot{f}, \ddot{f}, m_{1}, m_{2}=1.01 M_{\odot}, d=9.95 \mathrm{kpc}$.

to measure them; however, this is very unlikely to happen in the lifetime of eLISA (Stroeer \& Nelemans 2009).

Recent studies using EM data have shown that for the case of J0651, the period change can be enhanced by roughly up to 5\% due to tides (Burkart et al. 2013; Benacquista 2011; Piro 2011). Based on parameterized equilibrium tide theory, Piro (2011) has shown that for the J0651 system, in addition to GW radiation, the tidal interactions between the WDs will imprint a shift in the time of eclipses by $0.3 \mathrm{~s}$ after one year of timing. The dominant GW contribution advances the shift by $5.5 \mathrm{~s}$. Benacquista (2011) also calculated the deviation from the pure GR-driven inspiral, under the assumption that the WDs are tidally locked with the orbit and the GW radiation causes a small mismatch between the WD spin and orbital period. This causes a tidal distortion of the lower mass WD and assuming that this tidal energy is mostly transferred from the orbit to the spin keeping the system tidally locked, the tidal deviations were computed for J0651. Both of these works are corroborated by Burkart et al. (2013), who compute the tidal response of a J0651-like system assuming that both WDs are in resonance lock where the orbit and spin vary uniformly. It has been further claimed that for J0651, one should be able to detect the effect of tides in the GW phase shifts (Fuller \& Lai 2012). These results are based on modeling dynamical tides in a carbon-oxygen WD. The prospect of detecting such a phase shift in the GW data is very exciting as this could lead to measurements of the components' moments of inertia. However, in order for the tides to significantly shift in the collective phase of the GW signal, one needs to observe the system for millions of cycles according to the estimate of the evolution in the number of cycles only due to the tides (Equation (88), Fuller \& Lai 2012), which is not feasible with the currently planned eLISA mission.

In order to investigate the measurability of the abovementioned orbital parameters, we calculate the predicted GW uncertainties in those parameters as a function of orbital period. We summarize the data analysis and the selection of the binaries in Section 2. In Section 3, we estimate the expected EM uncertainties from mid-eclipse timing measurements. This is followed by a comparison of the accuracies from two types of measurements in Section 4. Finally, we summarize prospects of measuring deviation in evolution due to tides and the improvement in the knowledge of the WDs from combining the accuracies of GW and EM measurements from the measurement of the rate of change in the orbital period.

\section{2. eLISA BINARIES AND UNCERTAINTIES FROM THE GW DATA}

We obtain the GW accuracies by carrying out Fisher information matrix (FIM) calculations in order to determine whether the GW parameters $\dot{f}$ and $\ddot{f}$ can be measured over the two-year GW observations by the eLISA mission. We consider three binary systems for this purpose: the verification source J0651, a hypothetical high-mass J0651 system, and the highest $\dot{f}$ source we find in the population synthesis predictions (Nelemans et al. 2004). In the rest of the paper, we will refer to them as J0651 high-mass and high- $f$ systems respectively. We list the GW parameter values of all these systems in Table 1. For J0651 only $P_{\text {orb }}$ and $\dot{P}_{\text {orb }}$ are measured (Hermes et al. 2012). These have been converted to $f$, and $\ddot{f}$ with relations mentioned above. Since $\ddot{P}_{\text {orb }}$ is not yet measured, a fiducial $\ddot{f}$ has been chosen such that it agrees with the GR predictions. These values are slightly higher for the high-mass J0651 in accordance with the masses. For the high- $f$ system, the values $\dot{f}$ and $\ddot{f}$ are given by the simulation.

Our method and application of FIM to extract the GW parameter uncertainties has been described in detail in Shah et al. (2012). In this paper, we extend our previous FIM analyses to include nine GW parameters: dimensionless amplitude $(\mathcal{A})$, frequency $(f)$, polarization angle $(\psi)$, initial $\mathrm{GW}$ phase $\left(\phi_{0}\right)$, inclination $(\cos \iota)$, ecliptic latitude $(\sin \beta)$, ecliptic longitude $(\lambda)$, orbital decay rate $(\dot{f})$, and rate of change of that decay $(\ddot{f})$. Given these $(\mathrm{GW})$ parameters, we calculate a $9 \times 9$ FIM for all three systems. This implies not knowing any of the parameters a priori. By inverting this matrix, we get the variance-covariance matrix (VCM) which provides the uncertainties in the parameters and the correlations between them. We refer to our previous paper for the signal and noise modeling in computing the expected parameter uncertainties and the correlations between them. We list the full VCM matrices for J0651 and the high- $f$ systems in the Appendix, which include the normalized correlations between the nine parameters. The normalized correlations between parameters of J0651 and high $f$ are different because of the difference between their angular parameters (see Shah et al. 2013) and also due to their respective GW frequencies (Błaut 2011).

\section{UNCERTAINTIES FROM THE EM DATA}

In this section, we describe the prospects of extracting the uncertainties in $f, \dot{f}$, and $\ddot{f}$ from the electromagnetic data. J0651 has a measured $\dot{P}_{\text {orb }}=9.8 \pm 2.8 \times 10^{-12} \mathrm{~s} \mathrm{~s}^{-1}$ which is consistent with GR predictions (Hermes et al. 2012) within the error. The way this is typically measured is to compare the observed $(O)$ mid-eclipse times with computed $(C)$ values from a model with constant orbital period and fit the $O-C$ values as a function of time (e.g., Kepler et al. 1991). A possible resulting parabola gives evidence of a finite value of $\dot{P}_{\text {orb }}$ (Sterken 2005). The phase of the signal in cycles at an arbitrary time, $t$, after a 
reference time evolves and it is given by a Taylor expansion of the phase:

$$
\phi=\phi_{0}+f\left(t-t_{0}\right)+\frac{\dot{f}}{2}\left(t-t_{0}\right)^{2}+\frac{\ddot{f}}{6}\left(t-t_{0}\right)^{3}+\cdots,
$$

where $t_{0}$ is the epoch and $t$ is measured in the barycentric co-ordinates. As the source is observed for a longer time, the second and third terms gain significance. Given a duration of observation, $T_{\mathrm{obs}}$, and for a fixed resolution in phase $\left(\sigma_{\phi}\right)$, the uncertainties in the three orbital parameters can be estimated by (Mattox et al. 1998):

$$
\sigma_{f} \sim \frac{\sigma_{\phi}}{T_{\mathrm{obs}}} ; \quad \sigma_{\dot{f}} \sim 2 \frac{\sigma_{\phi}}{T_{\mathrm{obs}}{ }^{2}} ; \quad \sigma_{\ddot{f}} \sim 6 \frac{\sigma_{\phi}}{T_{\mathrm{obs}}{ }^{3}} .
$$

Considering an uncertainty of eclipse timing for J0651 of (Hermes et al. 2012) $\sigma_{T_{0}} \sim 0.725 \mathrm{~s}$ (see Table 2) results in a fractional phase error of $\sigma_{T_{0}} / P_{0} \sim 9.5 \times 10^{-4}$ turns. Assuming a constant phase error timing this source for a long time, for, e.g., $T_{\text {obs }} \sim 10 \mathrm{yr}$ using the above equation, we get, $\sigma_{f} \sim 10^{-12}$ $\mathrm{Hz}, \sigma_{\dot{f}} \sim 10^{-21} \mathrm{~Hz} \mathrm{~s}^{-1}$ and $\sigma_{\ddot{f}} \sim 10^{-30} \mathrm{~Hz} \mathrm{~s}^{-2}$. This implies that for $\mathrm{J} 0651$, the relative uncertainties are $\sigma_{\dot{f}} / \dot{f} \sim 10^{-5}$ and $\sigma_{\ddot{f}} / \ddot{f} \sim 6$. Thus timing J0651 will be very useful for pinning down the rate of change of frequency, however, the uncertainty in $\ddot{f}$ is very large. Below we will compare the uncertainties in decay rate and rate of decay for all three binaries using $\mathrm{GW}$ and EM observations for a range of orbital periods.

\section{MEASURABILITY OF $\dot{f}, \ddot{f}$}

A straightforward way to distinguish the tidal contribution from that of the GW radiation in the evolution of the binary is to measure the quantities $f, \dot{f}$, and $\ddot{f}$ with sufficient accuracy. The general relativistic predictions of the orbital decay in a binary orbit due to $\mathrm{GW}$ radiation alone gives the following relation (Webbink \& Han 1998):

$$
\left(\frac{\ddot{f} f}{\dot{f}^{2}}\right)_{\mathrm{GW}}:=y=\frac{11}{3},
$$

thus, a measure of any deviation from this numerical value measured within the parameter accuracies for detached binaries will provide a testbed for effects of the tides.

To get a rough estimate of the percentage of tidal contribution in the binary evolution of J0651-like systems, we can estimate Equation (3) for J0651 where the tidal contribution is taken into account since the individual masses and radii of this system have been measured from its light curve. This gives us an idea of what the uncertainties in $\dot{f}$ and $\ddot{f}$ must be in order to measure any deviation from the GR-driven binary evolution. Under the influence of GW radiation only, the rate of change of GW frequency changes according to

$$
\dot{f}_{0}=\frac{96 \pi}{5} \frac{G^{5 / 3}}{c^{5}}\left(\pi \mathcal{M}_{c}\right)^{5 / 3} f^{11 / 3},
$$

where $\mathcal{M}_{c}$ is the chirp mass given by:

$$
\mathcal{M}_{c}=\left(m_{1} m_{2}\right)^{3 / 5} /\left(m_{1}+m_{2}\right)^{1 / 5} \text {. }
$$

Including the contribution of tides and assuming that the WD spins are synchronized with the orbital period, the rate of change of orbital frequency changes according to (Benacquista 2011):

$$
\dot{f}=\dot{f}_{0}\left(1+5 \Delta_{Q}+3 \Delta_{I}\right)
$$

In the equation above,

$$
\Delta_{Q}=\frac{Q(\pi f)^{4 / 3}}{G^{2 / 3} M^{5 / 3}}, \quad \Delta_{I}=\frac{\left(I_{1}+I_{2}\right)(\pi f)^{4 / 3}}{\mu G^{2 / 3} M^{2 / 3}},
$$

where $Q=k_{2} I_{i}$ is the quadrupole moment, $k_{2}$ describes the structure of the star and $I_{i}=m_{1} r_{1}^{2}$ is the moment of inertia of each star (with radius $r_{i}$ ). This can be translated in terms of $P_{\text {orb }}, \omega$, or $f_{\mathrm{EM}}$ via: $\omega=2 \pi / P_{\text {orb }}=2 \pi f_{\mathrm{EM}}=\pi f$. Thus, including the orbital decay due to tides, the GR formulation in Equation (3) will then change according to:

$$
\left(\frac{\ddot{f} f}{\dot{f}^{2}}\right)_{\text {tides }+\mathrm{GW}}=\frac{\frac{11}{3}+25 \Delta Q+15 \Delta I}{1+5 \Delta Q+3 \Delta I} .
$$

Given the measured masses, radii, and the present orbital period (or equivalently GW $f$ ) of J0651 and the assumptions from (Benacquista 2011), we get $\left((\ddot{f} f) / \dot{f}^{2}\right)_{\text {tides+GW }}=3.73138$. This is a deviation from the GR-driven case of $11 / 3$ by only $1.7650 \% .^{5}$ In deriving this value, we only accounted for the lower mass WD which is distorted whereas the higher mass WD is relatively undistorted and thus its quadrupole moment can be ignored. The deviation above implies that the measured quantities from which $y$ is derived should have accuracies at the level of less than a few percent in order to distinguish tidal dissipation from $\mathrm{GW}$ radiation in $\mathrm{J} 0651$-like systems.

In Figure 1 fractional accuracies $f, \dot{f}$, and $\ddot{f}, y$ are plotted as a function of orbital period for the three binaries with $\mathrm{GW}$ parameter values listed in Table 1 . In the figure, the size of the open and filled circles and the square represent the $\mathrm{S} / \mathrm{N}$ of the system at that orbital period (or equivalently, the GW frequency) from the GW observations. These GW uncertainties decrease with increasing GW frequency as expected since they have higher $\mathrm{S} / \mathrm{Ns}$ and at high $f$ the resolution of the $\mathrm{GW}$ parameters decrease as Doppler modulation gains significance (see discussion in Shah et al. 2013; Cornish \& Larson 2003). The vertical lines in the top left panel from right to left are the lowest limit of the orbital periods of the high- $f$ system, highmass system, and J0651, respectively, where the mass transfer will ensue. This is derived simply by setting the Roche lobe of the donor WD (Eggleton 1983) equal to the size of its predicted zero-temperature radius from the mass (Verbunt \& Rappaport 1988). A more accurate estimate of the period at which mass transfer starts is obtained by fitting the spectra with the best matching He WD models and this gives a larger value for the $P_{\text {orb }}$, e.g., for J0651, the mass transfer will start when it evolves to a period of $\sim 420 \mathrm{~s}$ (Panei et al. 2007), making it difficult to disentangle the tidal effects. In the figure, the accuracies in the parameters from observing the EM timing measurements for J0651 are shown for an observation length of $10 \mathrm{yr}$ (diamonds) and for $20 \mathrm{yr}$ (pluses). The accuracy in $y$ for both the cases of GW and EM uncertainties is computed using propagation of errors using Equation (3). The timing accuracy is assumed to be constant for all periods and this implies that the uncertainties

\footnotetext{
5 This estimate depends strongly on the moment of inertia $I_{i}$ of each of the binary masses; in fact, the term $I_{2}$ (i.e., of the lower of the masses which is more tidally deformed by the more massive mass) derived from a model for a tidally deformed star is the term that most affects the ratio in Equation (8).
} 

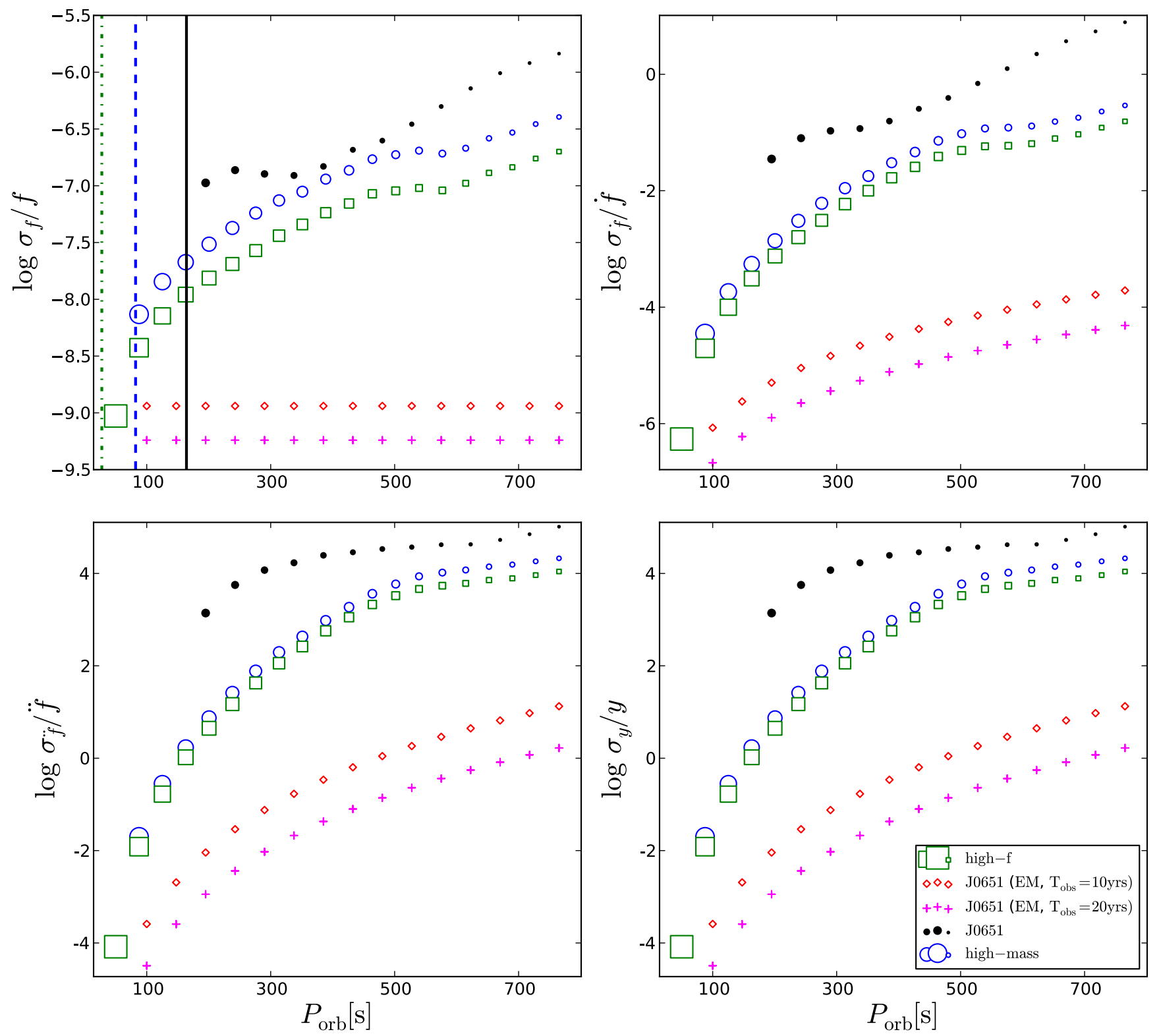

Figure 1. Relative uncertainties in frequency $(f)$, decay rate $(\dot{f})$, rate of decay rate $(\ddot{f})$, and braking index $(y)$ using gravitational wave data of J0651, the high-mass counterpart, and the high- $f$ binary. All the GW uncertainties are represented by (black) filled circles, (blue) open circles, and (green) squares for the three binary systems, respectively, listed above. Also the same uncertainties are shown for J0651 using its electromagnetic observations of its eclipse timings which are represented by (red) diamonds for $T_{\mathrm{obs}}=10 \mathrm{yr}$ and by (magenta) crosses for $T_{\mathrm{obs}}=20 \mathrm{yr}$. The marker sizes of filled/open circles and open squares represent the signal-to-noise ratio from the GW data of each system at that orbital period. The vertical lines in the top left panel are the values of minimum orbital period at which a given system will start mass transfer. The (black) solid line is for J0651, the (blue) dashed line is for the high-mass counterpart, and the (green) dash-dotted line is for the high- $f$ binary.

(A color version of this figure is available in the online journal.)

in the phase increase for smaller periods; however, the values of $\dot{f}$ and $\ddot{f}$ increase more steeply, thus we predict increasing accuracies of $\dot{f}$ and $\ddot{f}$ for smaller periods. It is clear from these uncertainties that measuring a tidal contribution from $\mathrm{GW}$ data alone is only possible if the contribution is huge for a system like J0651 before mass transfer starts. However, the EM and GW fractional uncertainties in $\dot{f}$ and $\ddot{f}$ for the high- $f$ binary are both very precise at $\sim 10^{-5}$ and $10^{-3}$, respectively, with which a small deviation in $y$ can be measured. However, the chances of observing an eclipsing high- $f$ binary within $1 \mathrm{kpc}$ is almost 0 , thus measuring tides for such a system only with EM is most likely not possible.

\section{COMBINING EM $\dot{f}$ AND GW MEASUREMENTS}

We find that from the timing measurements with $20 \mathrm{yr}$ duration, the orbital decay will be observed with fractional accuracies with up to five orders of magnitude better than the GW accuracy for a system like J0651. Coincidentally, a timing length of $20 \mathrm{yr}$ coincides with eLISA's launch, giving us an opportune time to combine the EM measurements with the GW ones to improve our knowledge of J0651-like system parameters. In this section, we address to what extent we can measure the tidal deviation terms introduced in Section 4. We also address how the knowledge of $\dot{f}$ improves the measurement 
of J0651's physical parameters of astrophysical interest such as the masses, inclination, and the distance to the source.

\subsection{Constraining the Tidal Deviation Terms, $\Delta_{Q}$ and $\Delta_{I}$}

The measurement of $\dot{f}$ can put constraints on the tidal contributions. Here we explore these constraints formulated in Benacquista (2011) which are expressed as $\Delta_{Q}$ and $\Delta_{I}$ in Equation (6). Under the same formulation, the GW amplitude which takes into account the quadrupole correction to the potential of the tidally distorted primary mass (less massive of the two) can be expressed as:

$$
\mathcal{A}=\mathcal{A}_{\mathrm{o}}(1+\Delta Q)
$$

where the GR-driven GW amplitude is given by:

$$
\mathcal{A}_{\mathrm{o}}=\frac{4\left(G \mathcal{M}_{c}\right)^{5 / 3}}{c^{4} d}(\pi f)^{2 / 3} .
$$

Assuming binary evolution is only driven by GWs, we compute the chirp mass in three ways for the J0651 system: (1) measurements of $m_{1}$ and $m_{2}\left(\mathcal{M}_{c}\right.$; Equation (5)), (2) measurements of $\mathcal{A}$ and $d\left(\mathcal{M}_{c}\left(\mathcal{A}_{0}, d\right)\right.$; Equations (9) and (10)), and (3) measurement of $\dot{f}\left(\mathcal{M}_{c}\left(\dot{f}_{\mathrm{o}}\right)\right.$; Equation (6)). The uncertainties in the measurements of $m_{1}, m_{2}$, and $d$ are taken to be $10 \%$ for the masses and distance. The uncertainty in the amplitude is taken from the FIM matrix $\sim 10 \%$ for an eclipsing J0651 and the uncertainty in $\dot{f}$ is taken to be $0.01 \%$, a conservative estimate from Figure 1. For the three estimates of chirp masses, we compare the values of $\Delta_{Q}$ and $\Delta_{I}$ for which the $\mathcal{M}_{c}\left(\mathcal{A}_{0}, d\right)$ and $\mathcal{M}_{c}\left(\dot{f}_{\mathrm{o}}\right)$ are inconsistent with $\mathcal{M}_{c}$. In the top panels of Figure 2, $\mathcal{M}_{c}$ is shown with a black line and the corresponding $1 \sigma$ uncertainty is shown by the (gray) hatched area. This estimate of $\mathcal{M}_{c}$ does not depend on $\Delta_{Q}, \Delta_{I}$. Since $\mathcal{M}_{c}(\dot{f})$ depends on both $\Delta_{Q}, \Delta_{I}$, it is plotted for two values of $\Delta Q=\left[10^{-5}, 0.12\right]$ shown by the upper and lower (blue) dashed lines, respectively, in the top left panel. In the top right panel, $\mathcal{M}_{c}(\dot{f})$ is shown for two values of $\Delta I=\left[10^{-5}, 0.068\right]$ corresponding to the upper and lower (blue) dashed lines, respectively. The predicted deviations from average measurements of the masses and radii for J0651 are marked by the (red) star. Benacquista (2011) estimate that $\Delta_{Q}, \Delta_{I}=1.46 \times 10^{-4}, 0.0166$ for $\mathrm{J} 0651$. The relative uncertainties of $\mathcal{M}_{c}(\dot{f})$ are at the level of $10^{-3}$, which is not visible in the figure. Finally, $\mathcal{M}_{c}(\mathcal{A}, d)$ can constrain $\Delta Q$ only and it is shown by the dotted line with uncertainties in the gray shaded area in the middle panel. The range of values of $\Delta_{Q}$ and $\Delta_{I}$ for which the three sets of chirp masses are inconsistent with each other within their uncertainties can be read from the figure; these are $\Delta_{I}>0.120$ and $\Delta_{Q}>-0.478$. In the bottom panel, the constraints on $\Delta_{Q}$ and $\Delta_{I}$ using both EM and GW data are shown by the (blue) dashed curve (via method 2) and the (dotted) vertical line (via method 3). From the bottom panel, it can be seen that measuring the tidal deviation $\Delta_{Q}$ is not feasible within the uncertainties in $\mathcal{A}, d$ marked by the (green) hatched area. Also measuring the deviation term $\Delta_{I}$, which is larger (at $\lesssim 10^{-2}$ ), is not feasible within the uncertainties in $m_{1}, m_{2}$ marked by the (gray) shaded area. Even though we expect strong tidal influence in detached WD systems such as J0651, measuring that contribution is unlikely unless the measurements in GW amplitude, distance, or the individual masses are also on the order of $\lesssim 10^{-2}$ for J0651-like systems. We conclude that tidal physics can be studied for high-mass binaries at opportune frequencies, which implies larger values of the decay rate measurable from the GW data.

\subsection{Constraining the Binary Parameters}

In our earlier work (Shah \& Nelemans 2014, hereafter SN201), we studied the effect of combining GW and EM observations where we considered the following EM measurements: the $d$ from the Gaia satellite, the primary mass $m_{1}$ from spectroscopy, the radial velocity $K_{1}$ also from spectroscopy, and possibly, the inclination $\iota$ from the fact that the binary could be eclipsing. We found that adding one or more of these measurements significantly improves our knowledge of the unknown astrophysical parameters of the binary and the improvement depends on the inclination of the source. In this study, we add the EM information of the orbital decay rate $\dot{P}_{\text {orb }} / \dot{f}$ (from Section 4 ) to the above list of EM observations and study whether and how much this improves the binary parameters' secondary mass $m_{2}$ and distance $d$ compared to scenarios considered in SN2013. The uncertainties in $m_{1}, K_{1}$, and $d$ are taken to be $10 \%$ as explained in SN2013, whereas $\dot{f}$ is taken to have an accuracy of $\sim 0.1 \%$ as measured from the timing eclipses of J0651 (see Section 4). Our method of combining each set of EM data with that of the GW data (i.e., amplitude $\mathcal{A}$ and inclination) is described in SN2013 and here we will summarize the advantage of including $\dot{P}_{\text {orb }}$ for each of the scenarios discussed in the earlier paper. Each of the scenarios below includes GW measurements $\mathcal{A}$ and $\iota$ of the J0651 system as a function of its inclination. We also assume the GW frequency of the source is known exactly since its relative uncertainty from the GW observation for the J0651 system is $10^{-7} \mathrm{~Hz}$.

1. Scenario 0: $G W$ data $+\dot{f}$ versus $G W$ data only. In SN2013 we found that distance can be estimated using GW amplitude. The chirp mass in this case was simply estimated for WDs using uniform distributions of the masses $\left(m_{i} \in[0.1,1.4] M_{\odot}\right)$ which are shown in gray in the top left panel of Figure 3. The 95th percentile in distances as a function of inclination are shown in the bottom left panel in gray. Adding EM data of $\dot{f}$ with $0.1 \%$ accuracy will constrain the 95 th percentile in $\mathcal{M}_{c}$ to a much better accuracy of $0.11 \%$ compared to the SN2013 which is shown in blue in the top left panel of Figure 3. The medians of these distributions are shown in solid lines and the real value is shown by the dashed black line. Hence the distances can be also constrained to much better accuracies shown in blue in the bottom left panel where we find that the (relative) 95th percentile uncertainties in distances range from $36 \%$ to $19 \%$ for inclinations of $5^{\circ}$ (face-on orientation) to $90^{\circ}$ (edge-on orientation) which are significantly better compared to the gray line found in SN2013.

2. Scenario 1: $G W$ data $+\dot{f}, d$ versus $\mathrm{GW}$ data $+d$. In SN2013, we found that using distance $d$ and $\mathcal{A}$ we could estimate $\mathcal{M}_{c}$ as a function of inclination where the 95th percentile in $\mathcal{M}_{c}$ fared better at higher inclinations with $\sim 16 \%$ and worse at lower inclinations. As shown above, adding EM data from $\dot{f}$ already constrains $\mathcal{M}_{c}$ much better to $0.11 \%$ for all inclinations. Thus, adding EM data on $d$ does not add much unless both $d$ and $\mathcal{A}$ are known to better accuracies than $\dot{f}$.

3. Scenario $2 a$ : $G W$ data $+\dot{f}, m_{1}$ versus $G W$ data $+m_{1}$. In SN2013, we found that combining EM data on $m_{1}$ with GW $\mathcal{A}$ provided an estimate of the secondary mass $m_{2}$ and constraints on the distance as a function of inclination. The distribution of $m_{2}$ (which is simply solved using the gray distribution in $\mathcal{M}_{c}$ in the top left panel) is shown also in gray 

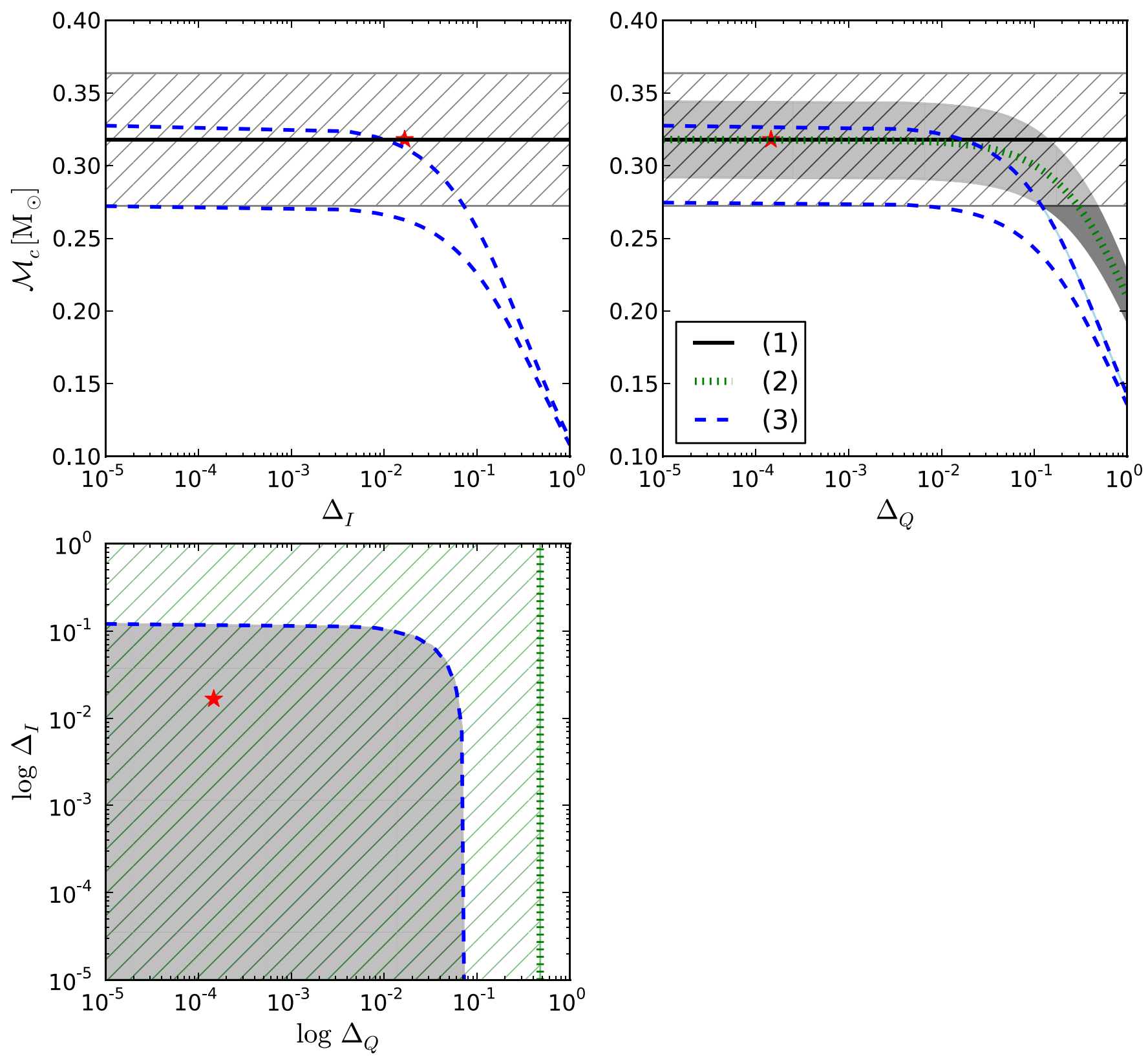

Figure 2. Measurability of tidal effects by determining the inconsistency between chirp mass $\mathcal{M}_{c}$ measurement from different methods as a function of the tidal deviation terms $\Delta_{I}$ and $\Delta_{Q}$ (characterizing the strength of the tides) for the J0651 system. The methods are: (1) using $m_{1}$ and $m_{2}$ in Equation (5), (2) using $\mathcal{A}$ and $d$ in Equations (9) and (10), and (3) using $\dot{f}$ in Equation (6). The top left and right panels show the $\mathcal{M}_{c}$ computed from method 1 (black solid line), from method 2 (green dotted line), and from method 3 (blue dashed lines). Method 1 is not influenced by tides (i.e., there is no dependence on $\Delta_{I}$, and $\Delta_{Q}$ ) whereas method 2 depends on $\Delta_{Q}$ only and method 3 depends on both $\Delta_{I}$ and $\Delta_{Q}$. The $1 \sigma$ uncertainties in the $\mathcal{M}_{c}$ for method 1 are from $\sigma_{m_{1}}, \sigma_{m_{2}}$ shown by the (gray) hatched area. For method 2 , they are from $\sigma_{\mathcal{A}}, \sigma_{d}$ shown by the gray filled area (in the top right of the panel). For method 3, the uncertainty from $\sigma_{\dot{f}}$ is not visible since the relative uncertainty is $\sim 10^{-3}$. In the top left panel, the top and bottom (blue) dashed curves correspond to two values of $\Delta_{Q}=10^{-5}, 0.0683$ and in the top right panel, the same correspond to two values of $\Delta_{I}=10^{-5}, 0.1205$ as the $\mathcal{M}_{c}$ measurement via $\dot{f}$ (method 3 ) depends on both tidal deviation terms. Observe that the three methods (in the top panels) show inconsistency in the chirp mass with increasing $\Delta_{I}, \Delta_{Q}$, however, the measurement uncertainties are too large in order to measure the inconsistency for small $\Delta_{I}, \Delta_{Q}$. The deviations at which the inconsistency can be measured within the uncertainties are determined by where the (blue) dashed lines and the (gray) shaded area cross with the (gray) hatched area in the top panels. In the bottom panel, these crossings are shown as a joint boundary in $\Delta_{I}$ and $\Delta_{Q}$. The estimated $\Delta_{I}$ and $\Delta_{Q}$ for the J0651 system are marked by the red star. It shows that constraining the tidal deviation terms is not feasible (for J0651-like systems) because typically the values of $\Delta_{I}$ and $\Delta_{Q}$ are smaller than the measurement uncertainties in $m_{1}, m_{2}, \mathcal{A}$, and $d$.

(A color version of this figure is available in the online journal.)

in the top middle panel of the figure. The 95 th percentiles in $d$ using this $m_{2}$ and $\mathcal{A}$ are shown in gray in the bottom middle panel. Adding EM data of $\dot{f}$ will improve the accuracy of $m_{2}$, reducing the 95 percentile uncertainty to $25 \%$ accuracy owing to a very accurate $\mathcal{M}_{c}$ (as discussed above). This reduced distribution in this $m_{2}$ is shown in blue in the top middle panel. This $m_{2}$, in combination with the GW $\mathcal{A}$, constrains the distance with better accuracies compared to SN2013 whose 95th percentiles are also shown as a function of inclination by the blue lines in the bottom left panel of the figure. Thus, adding $\dot{f}$ in this scenario improves $m_{2}$ and $d$ significantly. 

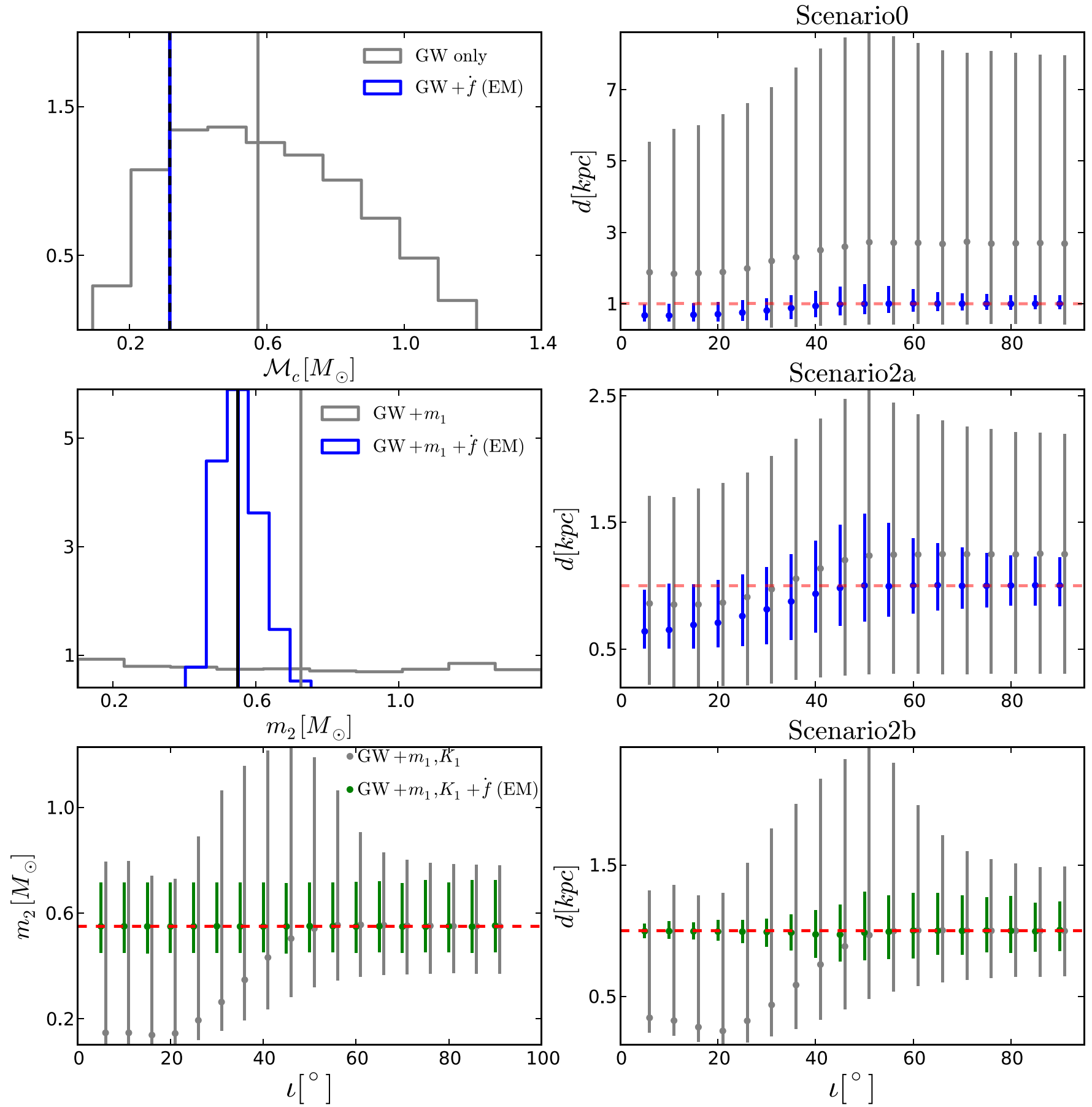

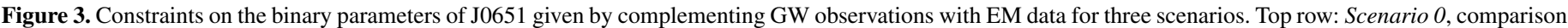

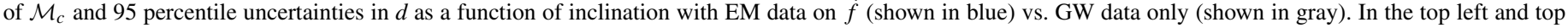

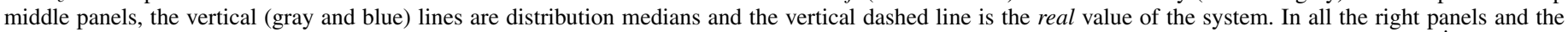

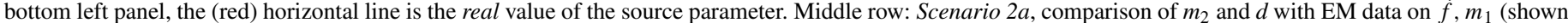

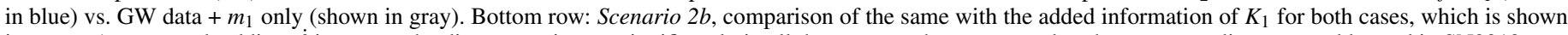
in green. As expected, adding $f$ improves the distance estimates significantly in all three cases when compared to the corresponding cases addressed in SN2013.

(A color version of this figure is available in the online journal.)

4. Scenario $2 b: G W$ data $+\dot{f}, m_{1}, K_{1}$ versus $G W$ data + $m_{1}, K_{1}$. In SN2013, we found that combining single-line spectroscopic data, i.e., $m_{1}, K_{1}$ with the $\mathrm{GW} \mathcal{A}$ constrained both the $m_{2}$ and $d$ as a function of inclination whose 95th percentiles are shown in gray in the top right and bottom right panels of Figure 3, respectively. Here we find that adding $\dot{f}$ will improve both of these quantities significantly; their respective 95th percentiles are shown with green lines. To explain these improvements we briefly explain how these quantities are estimated. As explained in the case above, we have an accurate constraint on $m_{2}$ using $m_{1}$ and $\mathcal{M}_{c}$. Using the $\mathrm{GW}$ inclination and the masses, we compute the radial velocity at each inclination, $K_{\mathrm{GW}}$. At each inclination, $K_{\mathrm{GW}}$ is compared with the measured $K_{1}$. 
Using the observed distribution, selecting a subset of $K_{\mathrm{GW}}$ with a probability distribution of $K_{1}$ constrains a subset in the rest of the parameters- $m_{1}, m_{2}, \mathcal{A}, d$-even further. The reduced uncertainties in $m_{2}, d$ calculated in this way are shown in green in the top right and bottom right panels of the figure. The method described is akin to Scenario 2c discussed in SN2013 in detail. Thus, adding EM data of $\dot{f}$ to $m_{1}, K_{1}$ improves the distance estimates significantly, especially at lower inclinations. We find the (relative) 95th percentile in $m_{2}$ is within the range of $25 \%-17 \%$ for faceon to edge-on systems. The same for $d$ is within the range of $6 \%-19 \%$.

5. Scenario 3: $G W$ data $+\dot{f}, m_{1,2}, K_{1,2}$ versus $G W$ data + $m_{1,2}, K_{1,2}$. In SN2013, we found that combining $m_{1,2}$ and $K_{1,2}$ with GW data improves the $\mathcal{A}$ and $\iota$ especially for lower inclination systems and this in turn constrains the distance of the binary (to roughly $30 \%$ ). Those distances can be compared with the independent estimate of the same using $\dot{f}$ explained above in Scenario 0 . Since $m_{1,2}$ are considered to have $10 \%$ accuracies much larger than $0.1 \%$ accuracy in $\dot{f}$, the chirp mass is still better determined in the case where $\dot{f}$ is known; thus, adding information from $m_{1,2}, K_{1,2}$ does not improve the constraint in distance any further.

\section{CONCLUSION}

We investigated the feasibility of detecting tides in detached (WD) binaries from the eLISA detector by calculating uncertainties of the parameters $\dot{f}$ and $\ddot{f}$ as a function of the orbital frequency. We implement Fisher matrix methods to compute the GW parameters' uncertainties and compare them with the accuracies from the mid-eclipsing timing measurements where the observation length is taken to be $\geqslant 10 \mathrm{yr}$. We also study the quantitative improvements in binary parameters when EM data on $\dot{P}_{\text {orb }}$ is combined with GW data and other possible sets of
EM data. From our analyses of J0651 and higher mass systems (see Table 1), we conclude:

1. Unless $e L I S A$ can discover systems like high- $f$ binaries, GW data alone will not suffice in measuring $\dot{f}$ and $\ddot{f}$ precisely enough for a system like J0651. However, finding such high- $f$ binaries near by $(\leqslant 1 \mathrm{kpc})$ is very unlikely.

2. Eclipse timing measurements for $10 \mathrm{yr}$ for J0651-like systems will provide a very precise measurement of $\dot{f}$ to less than $1 \%$. However, measuring a $2 \%-5 \%$ contribution from tides in $\dot{f}$ for such binaries is only possible if $\mathcal{M}_{c}$ and/or $d$ is also known to $\sim 1 \%$ accuracies. Additionally, detecting a collective phase shift in the GW phase using only GW data for J0651 as has been suggested (Fuller \& Lai 2012) is not possible.

3. For systems driven by only GW radiation, an EM measurement of $\dot{f}$ combined with a GW measurement of $\mathcal{A}$ provides us with a very precise measurement of $\mathcal{M}_{c}$. We compare this to our previous study in SN2013 where we computed improvement in binary parameters for the case of $\mathrm{J} 0651$ as a function of its inclination. We find that $\dot{f}$ can constrain $m_{2}$ and $d$ more accurately when considering various scenarios where EM data on $m_{1}, K_{1}$ are known. We find that knowing only $\dot{f}$ constrains the $1 \sigma$ in $\mathcal{M}_{c}$ to $0.3179 \pm 0.0002 M_{\odot}$. This further constrains $d$ from $0.70_{0.46}^{0.95} \mathrm{kpc}$ (face-on) to $1.00_{0.70}^{1.32} \mathrm{kpc}$ (edge-on). Adding EM data on $m_{1}$ constrains the $m_{2}$ to $0.55_{0.49}^{0.62} M_{\odot}$. Finally, adding EM data on $m_{1}, K_{1}$ constrains distance from $1.00_{0.96}^{1.03}$ $\mathrm{kpc}$ (face-on) to $1.00_{0.91}^{1.10} \mathrm{kpc}$ (edge-on). We conclude that compared to the scenarios in SN2013, our knowledge of the chirp mass, secondary mass, and distance will improve significantly when the eclipse timing measurements in $\dot{f}$ can be included in the GW-EM synergy.

This work was supported by funding from F.O.M.

\section{APPENDIX}

\section{VARIANCE-COVARIANCE MATRIXES OF J0651 AND B2}

We have listed the VCM matrices for the binary systems that we used in our analysis. There are nine parameters that described them and these are listed in the first row of the matrices below and for each binary, the values are listed in the row with $\theta_{i}$. The diagonal elements are the absolute uncertainties in each the nine parameters and the off-diagonal elements are the normalized correlations, i.e., $\mathrm{c}_{i i}=\sqrt{\mathcal{C}_{i i}} \equiv \sigma_{i}, \mathrm{c}_{i j}=\left(\mathcal{C}_{i j} / \sqrt{\mathcal{C}_{i i} \mathcal{C}_{j j}}\right)$. The strong correlations between parameters (i.e., whose magnitudes are 0.7$)$ are marked in bold in the VCMs below.

VCM 1: J0651, S/N 13.

\begin{tabular}{|c|c|c|c|c|c|c|c|c|c|}
\hline$\underline{\theta_{i}}$ & $\begin{array}{c}\mathcal{A} \\
1.67 \times 10^{-22}\end{array}$ & $\begin{array}{l}\phi_{0} \\
\pi\end{array}$ & $\begin{array}{l}\cos \iota \\
0.007\end{array}$ & $\begin{array}{c}f \\
2.61 \times 10^{-3}\end{array}$ & $\begin{array}{c}\dot{f} \\
-3.35 \times 10^{-17}\end{array}$ & $\begin{array}{c}\ddot{f} \\
1.57 \times 10^{-30}\end{array}$ & $\begin{array}{c}\psi \\
\pi / 2\end{array}$ & $\begin{array}{l}\sin \beta \\
0.01\end{array}$ & $\begin{array}{c}\lambda \\
1.77\end{array}$ \\
\hline $\begin{array}{l}\mathcal{A} \\
\phi_{0} \\
\cos \iota \\
f \\
\dot{f} \\
\ddot{f} \\
\psi \\
\sin \beta \\
\lambda\end{array}$ & $1.586 \times 10^{-23}$ & $\begin{array}{r}-0.0 \\
0.364\end{array}$ & $\begin{array}{c}0.0 \\
-0.01 \\
0.044\end{array}$ & $\begin{array}{c}0.01 \\
-\mathbf{0 . 9 1} \\
0.01 \\
3.807 \times 10^{-9}\end{array}$ & $\begin{array}{c}-0.01 \\
\mathbf{0 . 8 2} \\
-0.01 \\
-\mathbf{0 . 9 8} \\
1.059 \times 10^{-16}\end{array}$ & $\begin{array}{c}-0.0 \\
-0.01 \\
0.0 \\
0.01 \\
-0.04 \\
1.047 \times 10^{-26}\end{array}$ & $\begin{array}{l}0.02 \\
0.01 \\
-0.01 \\
-0.01 \\
0.01 \\
0.0 \\
0.041\end{array}$ & $\begin{array}{c}0.03 \\
0.11 \\
0.07 \\
-0.08 \\
0.04 \\
0.0 \\
-0.02 \\
0.069\end{array}$ & $\begin{array}{r}-0.06 \\
0.08 \\
-0.33 \\
-0.15 \\
0.19 \\
0.08 \\
0.05 \\
0.08 \\
0.019\end{array}$ \\
\hline
\end{tabular}


VCM 3: high-frequency binary, $\mathrm{S} / \mathrm{N} \sim 135$.

\begin{tabular}{|c|c|c|c|c|c|c|c|c|c|}
\hline$\underline{\theta_{i}}$ & $\begin{array}{c}\mathcal{A} \\
3.698 \times 10^{-22}\end{array}$ & $\begin{array}{l}\phi_{0} \\
3.666\end{array}$ & $\begin{array}{c}\cos \iota \\
-0.331 \\
\end{array}$ & $\begin{array}{c}f \\
17.695 \times 10^{-3}\end{array}$ & $\begin{array}{c}\dot{f} \\
1.988 \times 10^{-13}\end{array}$ & $\begin{array}{c}\ddot{f} \\
8.191 \times 10^{-24}\end{array}$ & $\begin{array}{c}\psi \\
1.97 \\
\end{array}$ & $\begin{array}{l}\sin \beta \\
0.685\end{array}$ & $\begin{array}{c}\lambda \\
5.411 \\
\end{array}$ \\
\hline $\mathcal{A}$ & $5.02 \times 10^{-24}$ & -0.15 & 0.79 & 0.05 & -0.05 & 0.05 & 0.28 & 0.29 & -0.21 \\
\hline$\phi_{0}$ & $(0.0136)$ & 0.048 & -0.07 & 0.87 & 0.82 & -0.76 & -0.36 & -0.26 & 0.02 \\
\hline $\cos \iota$ & & & 0.008 & 0.07 & -0.06 & 0.05 & 0.04 & 0.39 & -0.07 \\
\hline$f$ & & & & $8.228 \times 10^{-10}$ & -0.98 & 0.92 & -0.02 & 0.24 & 0.22 \\
\hline$\dot{f}$ & & & & $\left(4.65 \times 10^{-8}\right)$ & $5.169 \times 10^{-17}$ & -0.98 & -0.0 & -0.27 & -0.17 \\
\hline$\ddot{f}$ & & & & & $\left(2.6 \times 10^{-4}\right)$ & $1.4476 \times 10^{-24}$ & 0.02 & 0.30 & 0.14 \\
\hline$\psi$ & & & & & & $(0.176)$ & $9.86 \times 10^{-3}$ & -0.09 & -0.58 \\
\hline $\sin \beta$ & & & & & & & & $2.5 \times 10^{-4}$ & 0.14 \\
\hline$\lambda$ & & & & & & & & & $4.1 \times 10^{-4}$ \\
\hline
\end{tabular}

\section{REFERENCES}

Amaro-Seoane, P., Aoudia, S., Babak, S., et al. 2013, GWN, 6, 4 Benacquista, M. J. 2011, ApJL, 740, L54

Błaut, A. 2011, PhRvD, 83, 083006

Brown, W. R., Kilic, M., Hermes, J. J., et al. 2011, ApJL, 737, L23

Burkart, J., Quataert, E., Arras, P., \& Weinberg, N. N. 2013, MNRAS, 433,332

Cornish, N. J., \& Larson, S. L. 2003, CQGra, 20, 163

Cutler, C. 1998, PhRvD, 57, 7089

Dall'Osso, S., \& Rossi, E. M. 2013, MNRAS, 428, 518

Eggleton, P. P. 1983, ApJ, 268, 368

Fuller, J., \& Lai, D. 2012, MNRAS, 421, 426

Fuller, J., \& Lai, D. 2013, MNRAS, 430, 274

Hermes, J. J., Kilic, M., Brown, W. R., et al. 2012, ApJL, 757, L21

Kepler, S. O., Winget, D. E., Nather, R. E., et al. 1991, ApJL, 378, L45

Mattox, J. R., Halpern, J. P., \& Caraveo, P. A. 1998, ApJ, 493, 891
Nelemans, G., Yungelson, L. R., \& Portegies Zwart, S. F. 2004, MNRAS, 349,181

Panei, J. A., Althaus, L. G., Chen, X., \& Han, Z. 2007, MNRAS, 382,779

Piro, A. L. 2011, ApJL, 740, L53

Shah, S., \& Nelemans, G. 2014, ApJ, 790, 161 (SN201)

Shah, S., Nelemans, G., \& van der Sluys, M. 2013, A\&A, 553, A82

Shah, S., van der Sluys, M., \& Nelemans, G. 2012, A\&A, 544, A153

Sterken, C. 2005, in ASP Conf. Ser. 335, The Light-Time Effect in Astrophysics: Causes and Cures of the O-C Diagram, ed. C. Sterken (San Francisco, CA: ASP), 3

Stroeer, A., \& Nelemans, G. 2009, MNRAS, 400, L24

Verbunt, F., \& Rappaport, S. 1988, ApJ, 332, 193

Webbink, R. F., \& Han, Z. 1998, in AIP Conf. 456, Laser Interferometer Space Antenna, Second International LISA Symposium on the Detection and Observation of Gravitational Waves in Space, ed. W. M. Folkner (Melville, NY: AIP), 61 